\title{
Rules for Integrals over Products of Distributions from Coordinate Independence of Path Integrals
}

\author{
H. Kleinert* and A. Chervyakov ${ }^{\dagger}$ \\ Freie Universität Berlin \\ Institut für Theoretische Physik \\ Arnimallee 14, D-14195 Berlin
}

In perturbative calculations of quantum mechanical path integrals in curvilinear coordinates, one encounters Feynman diagrams involving multiple temporal integrals over products of distributions, which are mathematically undefined. In addition, there are terms proportional to powers $\delta(0)$ from the measure of path integration. We derive simple rules for their evaluation from the natural requirement of coordinate independence of the path integrals.

\section{INTRODUCTION}

While quantum mechanical path integrals in curvilinear coordinates can be defined uniquely and independently of the choice of coordinates within the time-sliced formalism [1], a perturbative definition on a continuous time axis poses problems. To exhibit the difficulties, consider the associated partition function calculated for periodic paths on the imaginary-time axis $\tau$ :

$$
Z=\int \mathcal{D} q(\tau) \sqrt{g} e^{-\mathcal{A}[q]},
$$

where $\mathcal{A}[q]$ is the euclidean action with the general form

$$
\mathcal{A}[q]=\int d \tau\left[\frac{1}{2} g_{\mu \nu}(q(\tau)) \dot{q}^{\mu}(\tau) \dot{q}^{\nu}(\tau)+V(q(\tau))\right] .
$$

The dots denote $\tau$-derivatives, $g_{\mu \nu}(q)$ is a metric, and $g=\operatorname{det} g$ its determinant. The path integral may formally be defined perturbatively as follows: The metric $g_{\mu \nu}(q)$ is expanded around some point $q_{0}^{\mu}$ in powers of $\delta q^{\mu} \equiv q^{\mu}-q_{0}^{\mu}$. The same thing is done with the potential $V(q)$. After this, the action $\mathcal{A}[q]$ is separated into a free part $\left.\mathcal{A}_{0}\left[q_{0} ; \delta q\right] \equiv \frac{1}{2} g_{\mu \nu}\left(q_{0}\right)\right) \dot{q}^{\mu} \dot{q}^{\nu}+\frac{1}{2} \omega^{2} \delta q^{\mu} \delta q^{\nu}$, and an interacting part $\mathcal{A}_{\text {int }}\left[q_{0} ; \delta q\right] \equiv \mathcal{A}[q]-\mathcal{A}_{0}\left[q_{0} ; \delta q\right]$.

A first problem is encountered in the square root in the functional integration measure in (11). Taking it into the exponent and expanding it in powers of $\delta q$ we define an effective action $\mathcal{A}_{\sqrt{g}}=-\frac{1}{2} \delta(0) \log \left[g\left(q_{0}+\delta q\right) / g\left(q_{0}\right)\right]$, which contains the $\delta$-function at the origin $\delta(0)$. It represents formally the inverse infinitesimal lattice spacing on the

\footnotetext{
${ }^{*}$ E-mail: kleinert@physik.fu-berlin.de

${ }^{\dagger}$ On leave from LCTA, JINR, Dubna, Russia E-mail: chervyak@physik.fu-berlin.de
}

time axis, and is equal to the infinite number $\delta(0) \equiv$ $\int d p /(2 \pi)$.

The second problem arises in the expansion of $Z$ in powers of the interaction, Performing all Wick contractions, $Z$ is expressed as a sum of loop diagrams. There are interaction terms involving $\dot{q}^{2} q^{n}$ which lead to Feynman integrals over products of distributions. The diagrams contain three types of lines representing the correlation functions

$$
\begin{aligned}
\Delta\left(\tau-\tau^{\prime}\right) & \equiv\left\langle q(\tau) q\left(\tau^{\prime}\right)\right\rangle=- \\
\partial_{\tau} \Delta\left(\tau-\tau^{\prime}\right) & \equiv\left\langle\dot{q}(\tau) q\left(\tau^{\prime}\right)\right\rangle=\cdots-.- \\
\partial_{\tau} \partial_{\tau^{\prime}} \Delta\left(\tau-\tau^{\prime}\right) & \equiv\left\langle\dot{q}(\tau) \dot{q}\left(\tau^{\prime}\right)\right\rangle=\ldots \ldots . .
\end{aligned}
$$

The right-hand sides define the line symbols to be used in Feynman diagrams for the interaction terms.

Explictly, the first correlation function reads

$$
\Delta\left(\tau, \tau^{\prime}\right)=\frac{1}{2 \omega} e^{-\omega\left|\tau-\tau^{\prime}\right|} .
$$

The second correlation function (4) has a discontinuity

$$
\partial_{\tau} \Delta\left(\tau, \tau^{\prime}\right)=-\frac{1}{2} \epsilon\left(\tau-\tau^{\prime}\right) e^{-\omega\left|\tau-\tau^{\prime}\right|}
$$

where

$$
\epsilon\left(\tau-\tau^{\prime}\right) \equiv-1+2 \int_{-\infty}^{\tau} d \tau^{\prime \prime} \delta\left(\tau^{\prime \prime}-\tau^{\prime}\right)
$$

is a distribution which vanishes at the origin and is equal to \pm 1 for positive and negative arguments, respectively. The third correlation function (5) contains a $\delta$-function:

$$
\partial_{\tau} \partial_{\tau^{\prime}} \Delta\left(\tau, \tau^{\prime}\right)=\delta\left(\tau-\tau^{\prime}\right)-\frac{\omega}{2} e^{-\omega\left|\tau-\tau^{\prime}\right|},
$$

The temporal integrals over products of such distributions are undefined [2].

In this note we define them uniquely by setting up rules between these and integrals over products of nonsingular correlation functions $\Delta\left(\tau-\tau^{\prime}\right)$, plus integrals over pure products of $\delta$-functions. These will be defined uniquely by the requirement of coordinate invariance of the path integral (11).

The internal consistency of these definitions is ensured by previous work of the present authors. In Ref. [3], we have shown that Feynman integrals in momentum space can be uniquely defined as $\epsilon \rightarrow 0$-limits of $1-\epsilon$ dimensional integrals via an analytic continuation à la 
't Hooft and M. Veltman [4]. This definition makes path integrals coordinate independent. In Ref. [5] we have given rules for calculating the same results directly from the Feynman integrals in the $1-\epsilon$-dimensional time space.

The present approach has the important advantage making superfluous the somewhat tedious analytic continuation to $1-\epsilon$ dimensions. In fact, it does not require specifying any regularization scheme. In addition, it gives a foundation of a new and general mathematics of extending the theory of distributions from a linear space to products.

\section{MODEL SYSTEM}

The announced derivation of the identities will be based on the requirement of coordinate independence of the exactly solvable path integral of a point particle of unit mass in a harmonic potential $\omega^{2} x^{2} / 2$, over a large imaginary-time interval $\beta$,

$$
Z_{\omega}=\int \mathcal{D} x(\tau) e^{-\mathcal{A}_{\omega}[x]}=e^{-\operatorname{Tr} \log \left(-\partial^{2}+\omega^{2}\right)}=e^{-\beta \omega / 2} .
$$

The action is

$$
\mathcal{A}_{\omega}=\frac{1}{2} \int d \tau\left[\dot{x}^{2}(\tau)+\omega^{2} x^{2}(\tau)\right]
$$

A coordinate transformation turns (10) into a path integral of the type (1) with a singular perturbation expansion. From our work in Refs. [3,5] we know that all terms in this expansion vanish in dimensional regularization. Here we shall require the vanishing to find the desired identities for integrals over products of distributions.

For simplicity we assume the coordinate transformation to preserve the symmetry $x \leftrightarrow-x$ of the initial oscillator, such its power series expansion starts out like $x(\tau)=f(q(\tau))=q-g q^{3} / 3+g^{2} a q^{5} / 5-\cdots$, where $g$ is a smallness parameter, and $a$ an extra parameter. We shall see that the identities are independent of $a$, such that $a$ will merely serve to check the calculations. The transformation changes the partition function (10) into

$$
Z=\int \mathcal{D} q(\tau) e^{-\mathcal{A}_{J}[q]} e^{-\mathcal{A}[q]}
$$

where is $\mathcal{A}[q]$ is the transformed action, whereas $\mathcal{A}_{J}[q]$ an effective action coming from the Jacobian of the coordinate transformation:

$$
\mathcal{A}_{J}[q]=-\delta(0) \int d \tau \log \frac{\delta f(q(\tau))}{\delta q(\tau)} .
$$

The transformed action is decomposed into a free part

$$
\mathcal{A}_{\omega}[q]=\frac{1}{2} \int d \tau\left[\dot{q}^{2}(\tau)+\omega^{2} q^{2}(\tau)\right]
$$

and an interacting part, which reads to second order in $g$ :

$$
\begin{aligned}
& \mathcal{A}_{\text {int }}[q]=\frac{1}{2} \int d \tau\left\{-g\left[2 \dot{q}^{2}(\tau) q^{2}(\tau)+\frac{2 \omega^{2}}{3} q^{4}(\tau)\right]\right. \\
& \left.+g^{2}\left[(1+2 a) \dot{q}^{2}(\tau) q^{4}(\tau)+\omega^{2}\left(\frac{1}{9}+\frac{2 a}{5}\right) q^{6}(\tau)\right]\right\}
\end{aligned}
$$

To the same order in $g$, the Jacobian action (13) is

$$
\mathcal{A}_{J}[q]=-\delta(0) \int d \tau\left[-g q^{2}(\tau)+g^{2}\left(a-\frac{1}{2}\right) q^{4}(\tau)\right] .
$$

For $g=0$, the transformed partition function (12) coincides with (10). When expanding $Z$ of Eq. (12) in powers of $g$, we obtain sums of Feynman diagrams contributing to each order $g^{n}$, which must vanish to ensure coordinate invariance. By considering only connected Feynman diagrams, we are dealing directly with the ground state energy.

\section{FREE ENERGY DENSITY}

The graphical expansion for the ground state energy will be carried here only up to three loops. At any order $g^{n}$, there exist different types Feynman diagrams with $L=n+1, n$, and $n-1$ number of loops coming from the interaction terms (15) and (16), respectively. The diagrams are composed of the three types of lines in (3)(5), and new interaction vertices for each power of $g$. The diagrams coming from the Jacobian action (16) are easily recognized by an accompanying power of $\delta(0)$.

At first order in $g$, there exists only three diagrams, two originated from the interaction (15), one from the Jacobian action (16):

$$
-g \bigcirc-g \omega^{2} \bigcirc+g \delta(0) \bigcirc \text {. }
$$

At order $g^{2}$, we distinguish several contributions. First there are two three-loop local diagrams coming from the interaction (15), and one two-loop local diagram from the Jacobian action (16):

$$
\begin{aligned}
& g^{2}\left[3\left(\frac{1}{2}+a\right)+15 \omega^{2}\left(\frac{1}{18}+\frac{a}{5}\right) \searrow\right. \\
& -3\left(a-\frac{1}{2}\right) \delta(0) \bigcirc
\end{aligned}
$$

We call a diagram local if it involves no temporal time integral. The Jacobian action (16) contributes further the nonlocal diagrams:

$$
-\frac{g^{2}}{2 !}\left\{2 \delta^{2}(0) \bigcirc-4 \delta(0)\left[\bigcirc+2 \omega^{4} \bigcirc\right]\right\} \text {. }
$$


The remaining diagrams come from the interaction (15) only. They are either of the three-bubble type, or of the watermelon type, each with all possible combinations of the three line types (3)-(5): The sum of all three-bubbles diagrams is

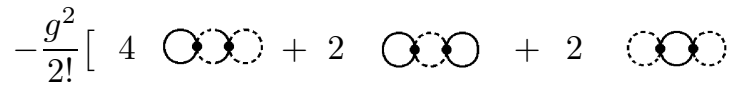

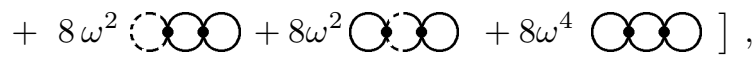

while the watermelon-like diagrams contribute

$$
-\frac{g^{2}}{2 !} 4\left[\Leftrightarrow+4 \Leftrightarrow+\Leftrightarrow+4 \omega^{2} \Longleftrightarrow+\frac{2}{3} \omega^{4} \supseteqq\right] \text {. }
$$

Since the equal-time expectation value $\langle\dot{q}(\tau) q(\tau)\rangle$ vanishes according to Eq. (7), there are a number of trivially vanishing diagrams, which have been omitted.

In our previous papers [3],5], all integrals were calculated individually in $D=1-\varepsilon$ dimensions, taking the limit $\varepsilon \rightarrow 0$ at the end. The results for the integrals ensured that the sum of all Feynman diagrams contributing to each order $g^{n}$ vanishes.

\section{RULES FOR INTEGRALS OVER DISTRIBUTIONS}

As a first step in calculating the Feynman integrals we express singular time derivatives $\dot{\Delta}(\tau), \ddot{\Delta}(\tau)$ in terms of regular correlation functions $\Delta(\tau)$, plus integrals over powers of $\delta$-functions. The tools for this will be partial integrations and the inhomogeneous field equation satisfied by the correlation function

$$
\ddot{\Delta}(\tau)=-\int d k \frac{k^{2}}{k^{2}+\omega^{2}} e^{i k \tau}=-\delta(\tau)+\omega^{2} \Delta(\tau),
$$

Most simply, we have for integrals over products of two correlation functions the relation

$$
\int d \tau\left[\dot{\Delta}^{2}(\tau)+\omega^{2} \Delta^{2}(\tau)\right]=\Delta(0)
$$

To prove this, we integrate the first term partially,

$$
\int d \tau \dot{\Delta}^{2}(\tau)=-\int d \tau \Delta(\tau) \ddot{\Delta}(\tau),
$$

with no boundary term due to the exponential vanishing at infinity of all functions involved. Using now the field equation (23) and the property of $\delta$-function that

$$
\int d \tau f(\tau) \delta(\tau)=f(0)
$$

for any smooth test function $f(\tau)$, we obtain (24).

We now turn to singular integrals involving $\Delta^{2}(\tau)$. Using the same tools, we obtain, in the same way, the relation $\int d \tau\left[\ddot{\Delta}^{2}(\tau)+2 \omega^{2} \dot{\Delta}^{2}(\tau)+\omega^{4} \Delta^{2}(\tau)\right]=\int d \tau \delta^{2}(\tau)$.

The last integral is undefined. Before fixing its value in the next section, we shall derive relations for integrals over singular products of four correlation functions. First for $\ddot{\Delta}(\tau) \Delta^{3}(\tau)$. Using again the field equation (23), we find

$$
-\int d \tau \ddot{\Delta}(\tau) \Delta^{3}(\tau)=\Delta^{3}(0)-\omega^{2} \int d \tau \Delta^{4}(\tau) .
$$

By a partial integration, the left-hand side becomes

$$
\int d \tau \ddot{\Delta}(\tau) \Delta^{3}(\tau)=-3 \int d \tau \dot{\Delta}^{2}(\tau) \Delta^{2}(\tau),
$$

leading to

$$
\int d \tau \dot{\Delta}^{2}(\tau) \Delta^{2}(\tau)=\frac{1}{3} \Delta^{3}(0)-\frac{1}{3} \omega^{2} \int d \tau \Delta^{4}(\tau) .
$$

Invoking once more the field equation (23), we obtain the integral

$$
\int d \tau \ddot{\Delta}(\tau) \dot{\Delta}^{2}(\tau) \Delta(\tau)=\omega^{2} \int d \tau \dot{\Delta}^{2}(\tau) \Delta^{2}(\tau),
$$

where we have used $\dot{\Delta}(0)=0$. Due to Eq. (30), this takes the form

$$
\int d \tau \ddot{\Delta}(\tau) \dot{\Delta}^{2}(\tau) \Delta(\tau)=\frac{1}{3} \omega^{2} \Delta^{3}(0)-\frac{1}{3} \omega^{4} \int d \tau \Delta^{4}(\tau) .
$$

A further partial integration reduces the integral

$$
\int d \tau \dot{\Delta}^{4}(\tau)=-3 \int d \tau \Delta(\tau) \dot{\Delta}^{2}(\tau) \ddot{\Delta}(\tau)
$$

to (32), such that we arrive at the relation

$$
\int d \tau \dot{\Delta}^{4}(\tau)=-\omega^{2} \Delta^{3}(0)+\omega^{4} \int d \tau \Delta^{4}(\tau) .
$$

We now consider an integral over $\ddot{\Delta}^{2}(\tau) \Delta^{2}$. Applying again the field equation (23), we find the relation

$$
\begin{aligned}
\int d \tau \ddot{\Delta}^{2}(\tau) \Delta^{2}(\tau) & =\int d \tau \Delta^{2}(\tau) \delta^{2}(\tau) \\
& -2 \omega^{2} \Delta^{3}(0)+\omega^{4} \int d \tau \Delta^{4}(\tau),
\end{aligned}
$$

The relations Eqs. (27) and (35) have reduced all integrals over singular products of correlation functions to regular integrals plus two undefined integrals containing $\delta^{2}(\tau)$. We are now going to show, that the reparametrization invariance of path integrals requires the following rules for integrals over products of two $\delta$-functions in Eqs. (27) and (35):

$$
\int d \tau \delta^{2}(\tau)=\delta(0)
$$

and further

$$
\int d \tau f(\tau) \delta^{2}(\tau)=f(0) \delta(0)
$$

for any smooth test function $f(\tau)$. 


\section{IMPOSING REPARAMETRIZATION INVARIANCE}

To first order in $g$, the sum of Feynman diagrams (17) must vanish:

$$
\bigcirc+\omega^{2} \bigcirc-\delta(0) \bigcirc=0
$$

The analytic form of this relation is

$$
\left[-\ddot{\Delta}(0)+\omega^{2} \Delta(0)-\delta(0)\right] \Delta(0)=0,
$$

and the vanishing is a direct consequence of the field equation (23) for the correlation function at origin.

At order $g^{2}$, the same equation reduces the sum of all local diagrams in (19) to a finite result plus a term proportional to $\delta(0)$ :

$$
\begin{aligned}
& {\left[-3\left(\frac{1}{2}+a\right) \ddot{\Delta}(0)+15\left(\frac{1}{18}+\frac{a}{5}\right) \omega^{2} \Delta(0)\right.} \\
& \left.-3\left(a-\frac{1}{2}\right) \delta(0)\right] \Delta^{2}(0)=\left[3 \delta(0)-\frac{2}{3} \omega^{2} \Delta(0)\right] \Delta^{2}(0) .
\end{aligned}
$$

Representing right-hand side diagrammatically, we obtain the identity

$$
\Sigma(19)=3 \delta(0) \bigcirc-\frac{2}{3} \omega^{2} \lesseqgtr 口,
$$

where $\Sigma(19)$ denotes the sum of all diagrams in Eq. (19). Using the identity (24) together with the field equation (23), we reduce the sum (20) of all one and two-loop bubbles diagrams to terms involving $\delta(0)$ and $\delta^{2}(0)$ :

$$
\begin{aligned}
& -\frac{1}{2 !}\left\{2 \delta^{2}(0) \int d \tau \Delta^{2}(\tau)\right. \\
& \left.-4 \delta(0) \int d \tau\left[\Delta(0) \dot{\Delta}^{2}(\tau)-\ddot{\Delta}(0) \Delta^{2}(\tau)+2 \omega^{2} \Delta(0) \Delta^{2}(\tau)\right]\right\} \\
& =2 \Delta^{2}(0) \delta(0)+\delta^{2}(0) \int d \tau \Delta^{2}(\tau) .
\end{aligned}
$$

Hence we find the diagrammatic identity

$$
-\frac{1}{2 !} \Sigma(20)=2 \delta(0) \bigcirc+\delta^{2}(0) \bigcirc \text {. }
$$

Now, the terms accompanying $\delta^{2}(0)$ turn out to be canceled by similar terms coming from the sum of all threeloop bubbles diagrams in (21). In fact, the identities (24) and (27) lead to

$$
\begin{aligned}
& -\frac{1}{2 !} \int d \tau\left[-4 \Delta(0) \ddot{\Delta}(0) \dot{\Delta}^{2}(\tau)+2 \Delta^{2}(0) \ddot{\Delta}^{2}(\tau)\right. \\
& \quad+2 \ddot{\Delta}^{2}(0) \Delta^{2}(\tau)+8 \omega^{2} \Delta^{2}(0) \dot{\Delta}^{2}(\tau) \\
& \left.\quad-8 \omega^{2} \Delta(0) \ddot{\Delta}(0) \Delta^{2}(\tau)+8 \omega^{4} \Delta^{2}(0) \Delta^{2}(\tau)\right] \\
& =-\left[\int d \tau \delta^{2}(\tau)+2 \delta(0)\right] \Delta^{2}(0)-\delta^{2}(0) \int d \tau \Delta^{2}(\tau) .
\end{aligned}
$$

Thus, we find the diagrammatic identity for all bubbles diagrams

$$
-\frac{1}{2 !} \Sigma(20)-\frac{1}{2 !} \Sigma(21)=-\int d \tau \delta^{2}(\tau) \bigcirc
$$

Finally, the relations (30), 35), 32) and (34) reduce the sum (22) of all watermelon-like diagrams to a finite contribution plus the integral involving $\delta^{2}(\tau)$ :

$$
\begin{aligned}
-\frac{4}{2 !} \int d \tau & {\left[\Delta^{2}(\tau) \ddot{\Delta}^{2}(\tau)+4 \Delta(\tau) \dot{\Delta}^{2}(\tau) \ddot{\Delta}(\tau)\right.} \\
+ & \left.\dot{\Delta}^{4}(\tau)+4 \omega^{2} \Delta^{2}(\tau) \dot{\Delta}^{2}(\tau)+\frac{2}{3} \omega^{4} \Delta^{4}(\tau)\right] \\
= & -2 \int d \tau \Delta^{2}(\tau) \delta^{2}(\tau)+\frac{2}{3} \omega^{2} \Delta^{3}(0)
\end{aligned}
$$

Combining these with all local diagrams (41), we easily verify that all finite contributions cancel each other leading to the diagrammatic identity

$$
\begin{aligned}
& \Sigma(19)-\frac{4}{2 !} \Sigma(22) \\
& \quad=\left[3 \delta(0)-2 \Delta^{-2}(0) \int d \tau \Delta^{2}(\tau) \delta^{2}(\tau)\right] \bigcirc .
\end{aligned}
$$

If the singular terms in Eqs. (45) and (47) are to sum up to zero, as required by the coordinate invariance of perturbatively defined path integrals, we must have the integration rules for the square distribution (36) and (37), which determined completely the right-hand sides of relations (27) and (35).

The procedure can easily be continued to higher-loop diagrams to obtain integrals over any desired products of singular correlation functions, and over products of $\delta$-functions.

At no place do we have to specify the value of $\delta(0)$ and the regularization scheme. There is a perfect cancellation of all powers of $\delta(0)$ arising from the expansion of the Jacobian action, and this is the reason why the so-called Veltman rule of setting $\delta(0)=0$ can be used everywhere without problems.

\section{SUMMARY}

In this note we have set up simple rules for relating singular to regular Feynman integrals which avoid the explicit calculation of dimensionally regularized integrals over products of distributions. These rules follow directly from the invariance of perturbatively defined path integral under coordinate transformations. Our procedure is independent of of regularization prescriptions, using only the fact that regularized integrals can be integrated by parts. The results are, of course, perfectly compatible with those derived before in Refs. [3,5] by dimensional regularization.

Just as in the time-sliced definition of path integrals in curved spcae in Ref. [1], there is absolutely no need 
for extra compensating potential terms found necessary in the treatments in Refs. [6 [8].

[1] H. Kleinert, Path Integrals in Quantum Mechanics, Statistics, and Polymer Physics, World Scientific, Singapore, 1995 (www.physik.fu-berlin.de/ ${ }^{\sim}$ kleinert/re.html\#b3).

[2] N.N. Bogoliubov and D.V. Shirkov, Introduction to the Theory of Quantized Fields, Interscience, New York, 1959.

[3] H. Kleinert and A. Chervyakov, Phys. Lett. B 464, 257 (1999) (hep-th/9906156).

[4] G. 't Hooft and M. Veltman, Nucl. Phys. B 44, 189 (1972).

[5] H. Kleinert and A. Chervyakov, Integrals over Distributions, and Reparametrization Invariance of Perturbatively Defined Path Integrals, quant-ph/9912056

[6] T.D. Lee and C.N. Yang, Phys. Rev. 128, 885 (1962).

[7] P. Salomonson, Nucl. Phys. B 121, 433 (1977).

[8] J. de Boer, B. Peeters, K. Skenderis and P. van Nieuwenhuizen, Nucl. Phys. B 446, 211 (1995); B 459, 631 (1996). 